\title{
Semiótica: Un recurso fundamental en los procesos de argumentación matemática escrita
}

\author{
Semiotics: A fundamental resource in the processes of written mathematical \\ argumentation
}

\section{Semiótica: Um recurso fundamental nos processos de argumentação matemática escrita}

\author{
Juan Pablo Salazar-Torres ${ }^{1}$, Yudith Liliana Contreras-Santander², Sandra Susana Jaimes-Mora ${ }^{3}$
}

Forma de citar: Salazar-Torres, J., Contreras-Santander, Y., \& Jaimes Mora, S. (2016) Semiótica: Un recurso fundamental en los procesos de argumentación matemática escrita. Revista Eco.Mat, 7, [20-32].

Recibido:

Mayo 4 de 2015

Aceptado:

Agosto 5 de 2015

\section{Resumen}

Todo proceso de argumentación matemática escrito exige un alto nivel de manejo de registros semióticos articulando estructuras semánticas capaces de significar. El presente artículo muestra los resultados del proyecto de investigación titulado "Análisis e interpretación de la semiótica en los procesos de argumentación matemática escrita por los estudiantes de $9^{\circ}$ grado del colegio Gonzalo Rivera Laguado de Cúcuta”, el estudio fue asumido desde las teorías en torno a la semiótica y argumentación matemática planteadas por Peirce, Bachelard, Vygotsky y Duval. La investigación se desarrolló bajo la metodología cuantitativa con un alcance descriptivo. Los resultados evidenciaron que en los procesos analizados de argumentación matemática escrita, se pierde fuerza y pertinencia del argumento debido al uso inapropiado de los recursos semióticos, evidenciando vacíos conceptuales que generan vacíos epistemológicos en el saber matemático.

Palabras clave: Argumentación matemática, lenguaje matemático, semiótica de la matemática.

${ }^{1}$ Magister en Educación j.salazar@unisimonbolivar.edu.co Orcid: 0000-0001-8285-2968

Universidad Simón Bolívar sede Cúcuta Cúcuta - Colombia

${ }^{2}$ Magister en Educación y.contreras@unisimonbolivar.edu.co Universidad Simón Bolívar sede Cúcuta

Cúcuta - Colombia

${ }^{3}$ Magister en Práctica Pedagógica sjaimes@unisimonbolivar.edu.co Universidad Simón Bolívar sede Cúcuta Cúcuta - Colombia

\begin{abstract}
Every process of written mathematical argumentation, requires a high level of management of semiotic records, articulating semantic structures capable of meaning. This manuscript shows the results of the research project entitled "Analysis and interpretation of semiotics in the processes of mathematical argumentation, written by the 9th grade students of the Gonzalo Rivera Laguado School in Cúcuta", the study was assumed from theories surrounding the semiotics and mathematical argumentation, proposed by Peirce, Bachelard, Vygotsky and Duval. The research was developed under the quantitative methodology with a descriptive scope. The results showed that in the analyzed processes of written mathematical argumentation, the argument loses its strength and relevance due to the inappropriate use of semiotic resources, evidencing conceptual gaps that generate epistemological gaps in mathematical knowledge.
\end{abstract}


Keywords: Mathematical argumentation, semiotics of mathematics, Language of mathematics.

\section{Resumo}

Todo processo de argumentação matemática escrito exige um alto nível de manejo de registros semióticos articulando estruturas semânticas capazes de significar. O presente artigo mostra os resultados do projeto de pesquisa titulado: "Análise e interpretação da semiótica nos processos de argumentação matemática escrita pelos estudantes de nona série do colégio Gonzalo Rivera Laguado de Cúcuta”, o estudo foi assumido desde as teorias em torno á semiótica e argumentação matemática levantadas por Peirce, Bachelard, Vygotsky e Duval. A pesquisa foi conduzida sob a metodologia quantitativa com alcance descritivo. Os resultados evidenciaram que nos processos analisados de argumentação matemática escrita, se perde força e pertinência do argumento devido ao uso inapropriado dos recursos semióticos, evidenciando vazios conceptuais que geram vazios epistemológicos no saber matemático.

Palavras-chave: Argumentação matemática, linguagem matemático, semiótica da matemática

\section{Introducción}

El lenguaje matemático emplea un conjunto de signos tanto orales como escritos que transmiten significados; el lenguaje humano tiene la capacidad de articular los signos formando estructuras complejas que adquieren una nueva capacidad de significación. La manera como hacemos sentido, como significamos, conocemos y construimos el contenido semántico de un signo, es a lo que Peirce (1974) llama "semiosis".

Desde esta misma lógica Alcalá (2009, p.21), afirma que "es evidente que el elemento específico del ser humano es su capacidad simbólica. Esta capacidad confiere la posibilidadderepresentarla realidad, valorarla, modularla virtualmente, transformarla y comunicar sus transformaciones y valoraciones, la construcción humana de significados se encuentra estrechamente ligada a la capacidad de simbolización". De esta manera, es en el contexto de lo matemático donde tiene lugar el uso y la interpretación de signos con carga de significados que permiten consolidar conceptos fundamentales para la representación y comprensión de la realidad.

En este sentido, es importante indagar sobre la problemática respecto a la aceptabilidad del argumento matemático que, según Duval (1999), se debe examinar desde los criterios de fuerza y pertinencia, criterios evidenciados en este caso, en situaciones problemas dentro de contextos matemáticos; dado que la fuerza de un argumento, según el mismo autor, depende del valor epistémico y éste se encuentra ligado a un estatuto teórico (hipótesis, teoremas, definiciones, etc.), el cual requiere unas representaciones semióticas, que en la argumentación escrita implica hacer uso de los diferentes signos, formando de esta manera un sistema semiótico capaz de significar y transformar una situación del lenguaje natural en lenguaje matemático.

Desde esta perspectiva reconocer la apropiación y uso del lenguaje matemático es totalmente necesario, ya que los signos 
No. 1

Enero-Diciembre 2016 ISSN 1794-8231 E-ISSN 2462 - 8794 PP: 20-32 utilizados en el proceso de comunicación matemática escrita son de una precisión rigurosa y deben estar libres de ambigüedades por cuanto el saber matemático está constituido por un cuerpo bien definido de signos, enunciados, conceptos y teoremas que requieren acudir a las reglas propias del lenguaje matemático.

Así, para el presente estudio se planteó como objetivo general analizar e interpretar las representaciones semióticas en los procesos de argumentación matemática escrita por los estudiantes del grado noveno del colegio Gonzalo Rivera Laguado del municipio de San José de Cúcuta. Para ello se realizó en un primer momento la identificación del uso y la interpretación que los estudiantes de éste grado tenían frente a los diferentes signos utilizados comúnmente en contextos matemáticos; en un segundo momento, se analizó y caracterizó la utilización de signos (símbolos, índices e íconos) inmersos en el proceso de la argumentación matemática escrita y finalmente se identificaron los errores semióticos asociados a la argumentación matemática escrita, proceso que se detalla desde lo metodológico más adelante.

Por otro lado, el horizonte teórico del estudio, estuvo fundamentado desde la teoría clásica de la semiótica de Peirce (1974), Puig (1994), la construcción de conceptos científicos de Vygotsky (1995), la teoría de los obstáculos epistemológicos de Bachelard (1976) y la estructura argumentativa propuesta por Duval (1999).

El trabajo se referencia desde antecedentes representativos en este campo, como son las investigaciones realizadas por León y Calderón (2001), cuyo propósito fue desarrollar una propuesta de tipo didáctico, que buscaba responder al problema basado en un estado generalizado de incompetencia comunicativa (lenguaje matemático) y matemática, en donde estructuraron cuatro requerimientos para desarrollar la competencia argumentativa en matemáticas; estos requerimientos fueron: el cognitivo, el epistemológico, el comunicativo y el sociocultural en el aula.

Asimismo, Ariza (2007) planteó la posibilidad de una elucidación de lo matemático desde una perspectiva derivada de las 'matemáticas en acción' y no desde una concepción 'analítico gramatical' de sus fundamentos y, estableció al menos, un mínimo umbral de validez que articulara una interpretación semiótica de los signos matemáticos, a través del análisis de un par de ejemplos elementales desde la geometría configuracional; este trabajo permitió un acercamiento a la comprensión de la teoría de Pierce desde el uso de la triada: índices, símbolos e íconos.

Otro trabajo de importante aporte a la investigación fue el trabajo de D'Amore (2006) resumido en su artículo Objetos, significados, representaciones y sentido, en el cual expuso que el uso de distintas representaciones y su progresiva articulación enriquecen el significado, el conocimiento, la comprensión del objeto, pero también su complejidad. El objeto matemático se presenta en cierto sentido como único, pero en otro sentido, como múltiple, entonces, ¿Cuál es la naturaleza del objeto matemático?, no parece que haya otra respuesta, que no sea la estructura formal, gramatical (en sentido epistemológico), y al mismo tiempo la estructura mental, global (en sentido psicológico) que los sujetos construimos en nuestros cerebros, a medida que crece nuestra experiencia.

La investigación se realizó en tres momentos; el primer momento se basó en el análisis descriptivo del uso que dieron los estudiantes a los diferentes signos matemáticos indagados desde el campo de función lineal; el segundo momento analizó los registros semióticos utilizados en el proceso argumentativo desarrollado por los estudiantes en una 
situación problema específica desde el contexto de la matemáticas $\mathrm{y}$, en el último momento, se identificaron los errores semióticos y conceptuales que describieron los estudiantes en dicho proceso argumentativo.

\section{Metodología}

La investigación se fundamentó desde la metodología cuantitativa con un diseño descriptivo, que de acuerdo con Hernández, Fernández y Baptista (2014, p.92), en éste diseño se "busca especificar propiedades y características importantes de cualquier fenómeno que se analice". Por ello, para la recolección de información, se aplicaron dos instrumentos de corte cuantitativo, una encuesta tipo test y una prueba tipo objetiva que presentaba una situación problema en contextos matemáticos, permitiendo realizar un análisis mediante la caracterización del uso de los signos (recursos semióticos) inmerso en un proceso de argumentación matemática escrita.

La población objeto de estudio fue de carácter finita, conformada por los estudiantes del colegio Gonzalo Rivera Laguado, contando con una muestra no probabilística intencionada, con un tamaño de veinte (20) estudiantes del grado noveno del colegio en mención, seleccionada bajo los criterios de interés del estudio según procesos académicos (estudiantes con alto y bajo rendimiento académico) y cognitivos frente al requerimiento de uso de niveles de pensamiento formal. Para Piaget (2001), los procesos cognitivos de pensamiento formal se ubican cronológicamente en los rangos de edades correspondientes a los estudiantes de grado 9 donde se da el uso de estructuras lógicas basadas en los esquemas u operaciones formales.

\section{Momentos de la investigación}

a. Primer Momento: Identificación del uso e interpretación de los signos matemáticos: Para esta etapa en la recolección de información se utilizaron como técnicas: la encuesta tipo test que buscaba realizar un diagnóstico del lenguaje matemático con el fin de identificar desde niveles cuantitativos el uso y la interpretación de los diferentes signos (según la caracterización de Pierce) utilizados comúnmente en situaciones matemáticas por los estudiantes del grado noveno; para ello la información se procesó recurriendo al análisis mediante la estadística descriptiva organizada en tablas de frecuencia y representada en gráficos estadísticos.

b. Segundo Momento: Análisis de la estructura argumentativa en una situación problema. Se aplicó una prueba tipo objetiva, que presentaba una situación problema en contextos matemáticos, buscando analizar y caracterizar el uso de signos (símbolos, índices e íconos) o registros semióticos utilizados en el proceso de argumentación matemática escrita.

c. Tercer momento: Identificación de errores semióticos asociados a la argumentación matemática. Se realizó un proceso de triangulación que permitió la confrontación de los hallazgos encontrados en los dos momentos anteriores, haciendo seguimiento sistemático a cada investigado, es decir, confrontando la respuesta de cada estudiante frente a los dos instrumentos aplicados, con el fin de establecer la incidencia frente a los errores conceptuales de los signos matemáticos en los procesos de argumentación al resolver la situación problema planteada.

\section{Resultados y discusión}

Como se expresó anteriormente, el análisis de la información desde el contexto de la semiótica (primer momento) se realizó
Enero-Diciembre 2016 ISSN 1794-8231 E-ISSN 2462 - 8794 PP: 20-32 
teniendo en cuenta la clasificación que realiza Pierce del signo: símbolos, índices e íconos (en su relación signo-objeto). En la tabla I, se representan los signos utilizados en el instrumento aplicado para el primer momento de la investigación. A continuación se muestra la operacionalización de la variable "Signos" que conformó el análisis de la semiótica lógica utilizado para éste primer instrumento.

Tabla I. Operacionalización de la variable signos de acuerdo a la semiótica lógica de Pierce.

\begin{tabular}{|c|c|c|c|}
\hline Objeto de estudio & Variable & Dimensiones & Subdimensiones \\
\hline \multirow{15}{*}{ Semiótica Lógica } & \multirow{15}{*}{ Signos } & Símbolos & Símbolos de operaciones básicas en matemáticas: +; -; $\mathrm{x} ; \div$ \\
\hline & & \multirow{4}{*}{ Índices } & Variables: $\mathrm{x}, \mathrm{y}, \mathrm{z}, \mathrm{m}, \mathrm{p}$, Etc. \\
\hline & & & Signos de agrupación: ()$;\{\} ;[]$ \\
\hline & & & Identificación de una línea recta: L1, L2 \\
\hline & & & Identificación de un punto de intersección entre dos restas: $\mathrm{P}(\mathrm{x}, \mathrm{y})$ \\
\hline & & \multirow{10}{*}{ Íconos } & Congruencia \\
\hline & & & Segmentos de rectas \\
\hline & & & Representación de una función F: $\mathrm{x}->\mathrm{y}$ \\
\hline & & & Expresiones matemáticas: $5 \mathrm{x} ;-4 \mathrm{y} ; 8 \mathrm{z}$ \\
\hline & & & Representación gráfica de líneas rectas \\
\hline & & & Signos de comparación: $>,<,=, \leq, \geq$ \\
\hline & & & Representación gráfica de un plano cartesiano \\
\hline & & & Ecuación general de una línea recta: $\mathrm{y}=\mathrm{mx}+\mathrm{b} ; \mathrm{f}(\mathrm{x})=\mathrm{mx}+\mathrm{b}$. \\
\hline & & & Ángulos: $\beta, \delta, \theta, \alpha, \gamma$ \\
\hline & & & Par ordenado $(\mathrm{x}, \mathrm{y})$ \\
\hline
\end{tabular}

Fuente: Autores.

4.1 Análisis y discusión de los símbolos.

Del análisis frente a los procesos de significación de los símbolos (ver figura 1), se encontró que el $20 \%$ de la fracción medida no contesto al instrumento, el $25 \%$ de los estudiantes significaron de manera correcta los símbolos y un 55\% de los estudiantes realizaron procesos de significación incorrecta.

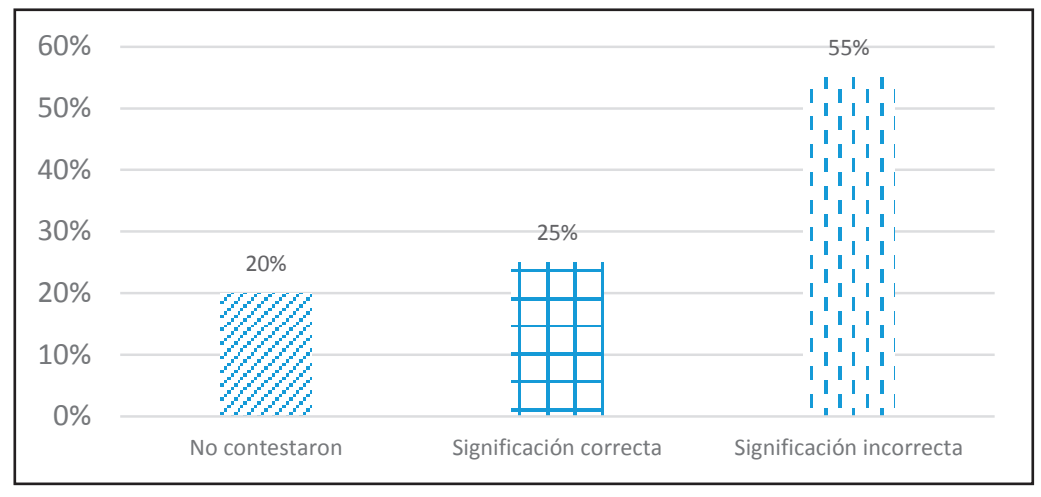

Figura 1. Resultados del proceso de significación de los símbolos. Fuente: Autores.

Para Pierce (1974), los símbolos dejan de significar sin el interpretante, es decir, es el sujeto quien le da sentido y significación a cualquier símbolo; así pues, dentro de esta afirmación que hace el autor emerge una palabra sencilla, pero de gran importancia en los procesos de las representaciones semióticas "significar", dependiendo del significado que se le asigne al signo se establece un represéntame (signo semejante al signo original), pero por otro lado el significado depende del sentido que el intérprete le otorgue al signo, tal como Vygotsky lo describe en su teoría de construcción de conceptos en el cual se define la relación: palabra (signo), sentido, significado y 
concepto ( representación semiótica). Desde esta perspectiva se evidenció que existe un bajo nivel en los estudiantes en cuanto a los procesos de significación, esta problemática es producida directamente por los procesos de sentido que el estudiante realiza con los símbolos dados, Peirce afirma que en los procesos de representaciones semióticas "existe una triple conexión del signo, la cosa significada y la cognición producida por la mente" (Pierce citado por Puig, 2003, $\mathrm{p}: 4)$, es decir, si el estudiante no realiza un proceso lógico entre el signo, el sentido del signo y su significado, es evidente que en las representaciones semióticas utilizadas en el proceso argumentativo (que para el caso es en el contexto de las matemáticas), se evidencian vacíos semióticos.

Este proceso tríadico al cual Peirce (1974) hace referencia, se denota claramente en los estudiantes que tuvieron argumentos sin fuerza, es decir, en aquellos que hicieron uso no apropiado del lenguaje matemático o de un signo en particular para argumentar el significado del símbolo (Marmolejo, 2012). Dentro de todo proceso argumentativo juega un papel fundamental los pre-conceptos del estudiante, Bachelard (1976, p:27) afirma que "en la formación del espíritu científico el primer obstáculo es la experiencia básica" o los conocimientos previos, es decir, que los individuos antes de iniciar cualquier estudio, tienen ya un conjunto de ideas acerca del cómo y el porqué de las cosas son como son, Bachelard (1976) acuña el término de "obstáculo epistemológico", al conocimiento erróneo producido a través de vacíos conceptuales, en donde los vacíos conceptuales son una consecuencia directa de los procesos de significado y sentido que realiza un estudiante cuando se enfrenta a un signo.

Durante el análisis de los símbolos se observó que, los errores conceptuales tienen una relación directa con los procesos de significación, donde el uso inapropiado de signos resultan ser vacíos conceptuales dentro de las construcciones semióticas en relación con los signos. Teniendo en cuenta la afirmación de Peirce (citado por Gorlée, $\mathrm{s} / \mathrm{f}, \mathrm{p} .10)$ "el símbolo está conectado con su objeto en virtud de la idea de la mente utilizadora de signos", se puede concluir que, el uso y buen manejo de los símbolos como recurso semiótico, exige un alto nivel de producción de sentido en los procesos de representaciones semióticas.

En términos generales se encontró que los estudiantes de 9 grado del colegio Gonzalo Rivera Laguado evidenciaron dificultades en los procesos de producción de sentido y significación de los símbolos, donde existen errores conceptuales tales como segmentos y signos de comparación generados por preconceptos erróneos y perjudicando la relación tríadica signo-objeto-signo que facilita los procesos de representaciones semióticas.

\subsection{Análisis y Discusión de los índices.}

La figura 2 muestra la descripción de los procesos de significación de los índices que conformaron el primer instrumento, siendo los índices de representación de rectas y puntos de intersección los que obtuvieron menos procesos de significación por los estudiantes (el 5\% en ambos signos); los signos variables y de agrupación mostraron los procesos de significación correctos con mayor porcentaje ( $10 \%$ y $20 \%$ respectivamente), sin embargo también tuvieron los porcentajes más altos de procesos de significación incorrecta $(50 \% \mathrm{y}$ $45 \%$ respectivamente).
Enero-Diciembre 2016 ISSN 1794-8231 E-ISSN 2462 - 8794 PP: 20-32 


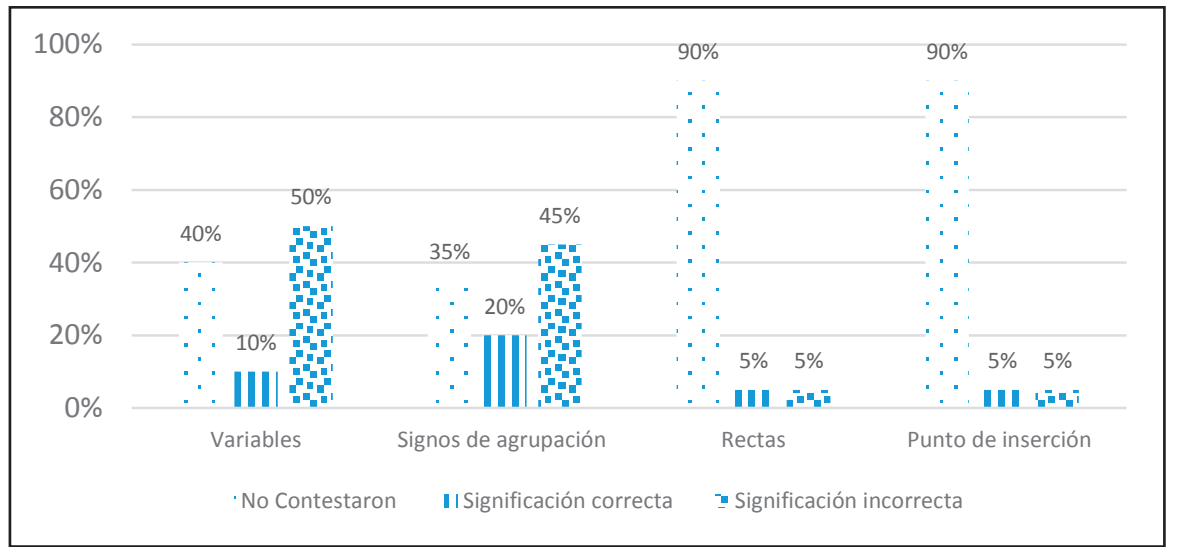

Figura 2. Resultados del proceso de significación de los índices. Fuente: Autores.

Para Pierce (citado por Vevia, 1997, p: 205), el índice "es un signo que tiene una conexión física directa con el objeto", el índice señala al objeto (signo inicial) que quiere representar, no se parecen a los objetos correspondientes y no describen el objeto. A demás Peirce (citado por Gorlée, s/f, p, 20) asegura que después de que esta relación (signo-objeto) está establecida, la mente interpretante, no tiene nada que ver con la conexión física entre el signo y el objeto.

Teniendo en cuenta la anterior consideración sobre los índices, se realizó el análisis de estos signos desde los resultados arrojados por el instrumento 1 (test), se utilizaron los índices más comunes dentro del campo temático de "función lineal"; los índices utilizados fueron: signos de comparación, variables, signos de identificación de rectas e identificación del punto de intercepción de dos rectas, en donde sólo el $40 \%$ de los estudiantes realizaron el proceso de significación correcto, estos procesos de significación como lo afirma Vygotsky, están relacionados directamente con los procesos de sentido que el estudiante le da al signo y, a su vez, refleja el uso apropiado del lenguaje matemático utilizado en sus argumentos para explicar cada índice en particular.

Se evidencia que en el $60 \%$ de los estudiantes de noveno grado, existe un nivel bajo en los procesos de sentido y significación que realizan de los índices; Así mismo se encontraron algunos errores conceptuales que están afectando el uso de recursos semióticos en los procesos de argumentación matemática dado por los estudiantes, estos errores conceptuales como lo afirma Bachelard, nacen de unos vacios conceptuales que tiene los estudiantes. Algunos de estos errores conceptuales fueron: rectas paralelas para el índice de identificación de rectas que está asociado con el índice punto de intersección de rectas cuyo vacío conceptual y uso inapropiado del lenguaje matemático fue punto de encuentro, así mismo los términos de separar y encerrar para el índice de signos de agrupación y los términos de letras y símbolos para el índice de variables.

Para los signos de agrupación y las variables, se encontró respectivamente que el $90 \%$ y el $80 \%$ de estudiantes no emplearon un término apropiado (lenguaje matemático) para escribir el nombre que le daban al signo, es decir, recurren al lenguaje natural y no al lenguaje matemático o utilizan un lenguaje matemático no apropiado para representar el nombre de un signo, causal de la no contextualización del saber matemático.

De manera global se puede afirmar que esta dificultad (uso no apropiado del lenguaje matemático) se manifiesta específicamente 
en los procesos de argumentación escrita, en el momento de significar, definir o representar un signo, así mismo se detectaron los errores conceptuales que nacen de los vacíos conceptuales que están afectando las representaciones semióticas en los estudiantes de noveno grado del Colegio Gonzalo Rivera Laguado.

\subsection{Análisis y discusión de los íconos}

La figura 3 muestra los resultados descriptivos de los procesos de significación que realizaron los estudiantes frente a los íconos. El signo "ángulos", fue el que mejor significaron los estudiantes (un 35\% de la fracción evaluada), sin embargo la gran mayoría de los estudiantes no realizaron procesos de significación y otros estudiantes aunque realizaron dicho proceso, presentaron errores de tipo semiótico, siendo los signos de congruencia y comparación con el mayor porcentaje frente a los procesos de significación incorrecta (55\% y $\quad 45 \%$ respectivamente).

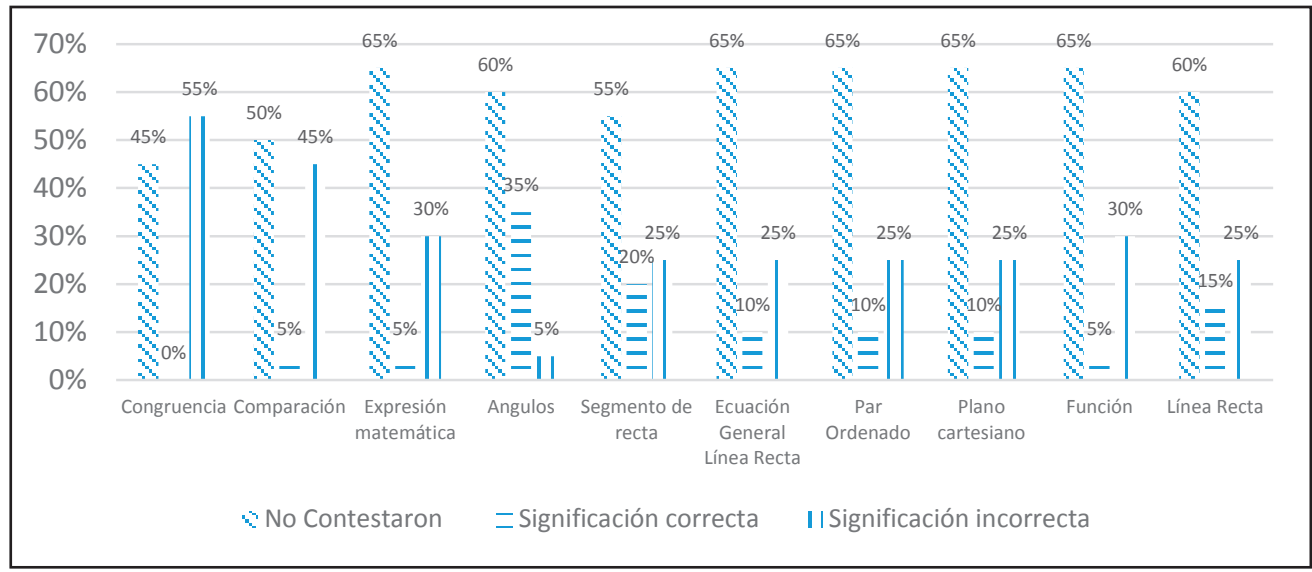

Figura 3. Resultados del proceso de significación de los Iconos.

Fuente: Autores.

En la clasificación que hace Peirce de los signos en su teoría semiótica, además de los símbolos e índices, se encuentran los íconos, para Peirce (citado por Vevia, 1997), los íconos "tienen el carácter que los hace significar incluso si el objeto no existiera" (p.205); a diferencia de los índices, los íconos no tienen una relación directa con el objeto, así mismo afirma Peirce (1974) "los íconos son semejantes al objeto que representan" (p.205), es decir, el contenido semántico del objeto e ícono son semejantes, dicho de otra forma, hay una relación racional entre el ícono y el objeto.

Dentro de esta conexión racional entre el objeto y la mente interprete, se tiene en cuenta según De Lorenzo (1989), dos tipos de sentido del signo, el sentido eidético que se refiere al contenido semántico del signo, y el sentido operacional en el sentido que se sabe dónde se puede utilizar, así mismo, los procesos de significación de los íconos depende de la capacidad racional (sentido), del contexto matemático y de los conocimientos previos que el estudiante tenga en relación al signo, de igual forma existe una relación cognitiva entre el contenido semántico del signo y la
Enero-Diciembre 2016 ISSN 1794-8231 E-ISSN 2462 - 8794 PP: 20-32 capacidad operacional del signo.

Si analizamos el contexto general de la actividad matemática, se encontrará que los íconos ya están globalmente definidos, por ejemplo el signo " $y=m x+b$ ", es una la de las representaciones semióticas de una línea recta, independientemente cual sea la recta, se sabe que es una línea recta que corta en la coordenada $b$ del eje " $y$ " y que tiene una 
No. 1 pendiente de valor $\mathrm{m}$, lo anterior es a lo que De Lorenzo (1989) llama contenido semántico del signo, y ese contenido semántico nace de los procesos de sentido y significación que se hacen en relación al signo, este contenido semántico evidencia el lenguaje matemático, los preconceptos y los errores conceptuales ( $\mathrm{si}$ existen), además dependiendo de lo anterior, el ícono es pertinente o no en los procesos de argumentación matemática.

En este sentido, para los Iconos de congruencia y signos de comparación, se evidencio que no existe un lenguaje matemático apropiado para significar los íconos: por ejemplo para los estudiantes, el signo de congruencia " $\approx$ " tiene el mismo sentido del signo igual "=”, existe allí un error conceptual, es evidente que el contenido semántico del signo no está claro, lo que perjudica la relación racional entre el signo y el interpretante.

Así mismo, para los Iconos "expresiones matemáticas” y "ángulos”, se evidenciaron errores conceptuales dentro de las expresiones matemáticas y uso inapropiado del lenguaje matemático; por ejemplo, algunos estudiantes utilizaron términos inapropiados para representar el nombre de las expresiones matemáticas como números, ecuaciones, numero con incógnita y variables, si analizamos el contenido semántico para todos estos términos son muy diferentes (aunque exista una relación próxima entre todos ellos), sin embargo para los estudiantes tienen el mismo sentido o son equivalentes estos signos. Al hablar de errores conceptuales, es claro que existen vacíos conceptuales en los estudiantes y que están dificultando los procesos de significación en los registros semióticos dados en el procesos de argumentación matemática por los estudiantes de noveno grado.

Por otro lado, para los Iconos " $y=m x+b$ " y segmentos, se detectó que los estudiantes confunden el ícono de segmento con el ícono de punto y en el icono de " $y=m x$ $+b$ ", los estudiantes realizaron una escritura en lenguaje natural (no hicieron uso del lenguaje matemático), además la mayoría de los estudiantes no realizaron procesos de significación correctos, estos debido a los errores conceptuales que presentan en relación a estos signos.

De igual forma, para los íconos “ $(x, y)$ ” $\mathrm{y}$ representación gráfica del plano cartesiano, se estableció la relación entre los procesos de significación y los errores conceptuales, por ejemplo para el ícono “ $(x, y)$ ”, se encontró que los estudiantes tienen varios errores conceptuales al establecer un nombre para el ícono, estos vacíos fueron, letras, pendiente y dos puntos, en el momento que el estudiante realiza el registro semiótico de letras, se evidencia claramente que no existe una contextualización en el campo matemático, y esta contextualización no se da, debido a que no se realiza los procesos de sentido y significación del signo, por otro lado la relación racional entre el estudiante y el signo afectarán los procesos de representaciones semióticas en el campo de la matemáticas, afectando los procesos de argumentación matemática; en esta perspectiva León y Calderón, (2001, p: 39) afirman que "la situación argumentativa exige, no sólo la producción de argumentos (registros semióticos, término o signo), sino también, la producción de tramas argumentativas; es decir, de un discurso que además de estar conformado por argumentos, evidencie una intencionalidad y una conexión de los mismos, como efecto del funcionamiento de los sistemas semióticos".

Por último, para los Iconos " $f=x \rightarrow y$ " $\mathrm{y}$ representación gráfica de líneas rectas, los errores conceptuales que se encontraron fueron: para el primer ícono " $f=x \rightarrow y$ ", por ejemplo, algunos estudiantes utilizaron el término fórmula para representar el signo "f $=x \rightarrow y "$.

En términos generales se puede afirmar que, 
el contenido semántico de los signos se ve afectado por el uso inapropiado del lenguaje matemático, los errores conceptuales y la no contextualización del saber matemático, afectando el contenido semántico del sentido eidético del signo, lo cual afectará la relación tríadica (s-o-s) del campo semiótico dentro de los procesos de argumentación matemática. Lo anterior implica que, la utilización de los registros semióticos sea errónea y no pertinente para las construcciones argumentativas desarrolladas por los estudiantes de noveno grado del colegio Gonzalo Rivera Laguado.

\subsection{Uso de la semiótica en el proceso de argumentación matemática escrita.}

Como se mencionó anteriormente, para el segundo momento se aplicó una prueba objetiva compuesta por una situación problema, en el cual, a los estudiantes, se le realizaron dos preguntas concretas sobre la situación, estas dos preguntas buscaban de cierta manera que el estudiante a través de procesos matemáticos y haciendo uso de la semiótica, argumentara desde el contexto de la matemática la solución a las preguntas planteadas.
Del análisis se determinó que ningún estudiante completó los procesos matemáticos que le ayudaban a encontrar la solución a las preguntas; se evidenció que los signos utilizados por los estudiantes dentro de estos procesos matemáticos estuvo relacionados con las expresiones matemáticas, variables, símbolos de operaciones, y las dos ecuaciones lineales, ésta última fue el registro semiótico utilizado para representar las avenidas vecinales de la situación problema.

Para Peirce, la utilidad de las fórmulas algebraicas consiste precisamente en esa capacidad de develar verdades imprevistas, en este sentido, se encontró que en el último signo utilizado por los estudiantes (ecuación lineal), se evidenciaron problemas de sintaxis (ver tabla II) que afectaron el contenido semántico del ícono (ecuación lineal). Así mismo el $25 \%$ de los estudiantes hicieron uso del ícono plano cartesiano, el 15\% de los estudiantes del ícono par ordenado y sólo el 5\% de los estudiantes realizó una representación gráfica del ícono línea recta.

Tabla II: Errores semánticos del ícono en algunos estudiantes.

\begin{tabular}{|l|c|}
\hline \multicolumn{1}{|c|}{ ESTUDIANTES } & SIGNO UTILIZADO POR EL ESTUDIANTE \\
\hline ESTUDIANTE A & $\begin{array}{c}x+3 y=18-3 y=18 \\
x-1+2 y=-2\end{array}$ \\
\hline ESTUDIANTE B & $\begin{array}{c}x=2 x+3 x=18 \\
\end{array}$ \\
ESTUDIANTE C & $2 x+3 y=18-3 y \quad x=\frac{18-3 y}{2}$ \\
& $x+2 y=-2+x \quad y=\frac{-2+x}{2}$ \\
& $y=\frac{2-x}{2}=1$ \\
ESTUDIANTE D & $2 x+4 y=18 x$ \\
\hline
\end{tabular}

Fuente: Autores. Casos concretos del instrumento 2.

Por otro lado se evidenció dentro de los procesos matemáticos utilizados por los estudiantes, que no hay procesos claros para dar solución a un sistema lineal, así mismo se observó que habían procesos que no eran pertinentes dentro del contexto del proceso matemático. Se encontró que los estudiantes de noveno grado del colegio Gonzalo Rivera Laguado, no desarrollan procesos adecuados de sentido y significación de los signos. Así mismo durante el análisis de la prueba objetiva, se evidenció problemas en cuanto al sentido operacional del signo, en donde los estudiantes utilizaban signos
Enero-Diciembre 2016 ISSN 1794-8231 E-ISSN 2462 - 8794 PP: $20-32$ 
No. 1

Enero-Diciembre 2016 ISSN 1794-8231

E-ISSN 2462 - 8794 PP: 20-32 correctos pero en situaciones no pertinentes. Lo anterior llevo a que el uso no apropiado del lenguaje matemático y los errores conceptuales encontrados durante los análisis de la investigación, resultan ser obstáculos del proceso argumentativo en contextos matemáticos desarrollados por los estudiantes.

Según Duval (1999), la argumentación en sentido amplio tiene como finalidad hacer cambiar el valor epistémico semántico de una proposición, modificando el grado de convicción de un interlocutor, para que acepte o rechace la proposición, basados en esta afirmación de Duval se encuentra el primer problema que resulta en los estudiantes, si retomamos un fragmento de lo anterior la argumentación en sentido amplio tiene como finalidad hacer cambiar el valor epistémico semántico de una proposición, se encuentra que, como los estudiantes poseen varios errores conceptuales de los signos, es claro que el contenido semántico de los signos utilizados por los estudiantes no es el apropiado.

Se evidencio durante los análisis de la investigación, que los estudiantes no le daban el sentido apropiado al signo, afectando así el significado del signo $\mathrm{y}$ el sentido operacional del mismo, en esta perspectiva para Vigotsky el sentido de una palabra (signo), es el agregado de todos los hechos psicológicos emergentes en nuestra conciencia a causa de esta palabra. Por lo tanto, el sentido de esta palabra (signo) siempre resulta ser una formación dinámica, esta formación dinámica, es a lo que Peirce le llama conexión tríadica Signo-objetosigno (S-O-S), para Peirce existe una triple conexión del signo, la cosa significada y la cognición producida por la mente, donde lo anterior juega un papel fundamental en la relación sentido-significación del signo a la cual se refiere Vigotsky.

\subsection{Semiótica y argumentación.}

Durante esta investigación en los diferentes análisis teóricos se deja claro la importancia que tiene la semiótica en los contextos de argumentación matemática, el uso apropiado del registro semiótico, como se ha determinado en este trabajo, depende de varios factores, entre los que se resaltan; los factores cognitivos, factores cognoscitivos, $\mathrm{y}$ factores sociales, estos entendidos desde las teorías de Vigotsky, De Lorenzo, Pierce, Bachelard y Duval.

Es necesario destacar la importancia y necesidad de uso apropiado del lenguaje matemático dentro del contenido semántico de un argumento (que compone un proceso argumentativo), solo así se puede consolidar la fuerza de los argumentos, según Duval (1999, s.p) "el valor y la fuerza de un argumento depende del dominio de conocimientos $y$ del contexto particular que da sentido a la situación argumentativa y motiva el recurso a los argumentos", Asimismo afirma Duval (1999, s.p) que "la fuerza depende principalmente de su adaptación a la situación, puesto que en matemática lo que está en juego son las restricciones propias del problema a resolver, luego estas restricciones determinan la elección de los argumentos donde es necesaria una serie de operaciones sucesivas que ponen en funcionamiento un sistema semiótico", lo anterior implica que los estudiantes tengan un buen desarrollo en los procesos argumentativos; finalmente Duval (1999, p.7) afirma que "un argumento se acepta o se rechaza con respecto a dos criterios: su pertinencia y su fuerza"; Fuerte, en el sentido que resista a contraargumentos y tenga un valor epistémico valido positivo $\mathrm{y}$ pertinente en cuanto sus argumentos tenga una conexión semántica lógica. 


\section{Conclusiones}

Los estudiantes del grado noveno evidencian errores conceptuales en el momento de definir, explicar o argumentar un signo, afectando la relación racional con los íconos, dado que estos tienen el carácter de significar, incluso si el objeto no existiera, además presentan problemas en la relación tríadica $(\mathrm{S}-\mathrm{O}-\mathrm{S})$ con los índices, en donde los índices dejan de significar sin el objeto porque existe una relación física directa, y problemas del proceso sentido-significación con los símbolos, donde los símbolos dejan de significar sin que exista la mente utilizadora de signos (interprete).

Los estudiantes del grado noveno del Colegio Gonzalo Rivera Laguado, no desarrollan procesos adecuados de sentido y significación de los signos (íconos, índices y símbolos), en donde se encontraron problemas del sentido eidético y del sentido operacional del signo, este último, reflejándose claramente en los resultados del instrumento 2 , de igual forma se puede afirmar que el problema eidético (contenido semántico del signo) que tienen los signos, nacen del uso no apropiado del lenguaje matemático y de los errores conceptuales que poseen dichos estudiantes.

El uso no apropiado del lenguaje matemático y los errores conceptuales que se encontraron en los estudiantes del colegio Gonzalo Rivera Laguado en relación a los signos, están afectando la fuerza y la pertinencia de los argumentos dificultando los procesos de argumentación matemática.

La utilización de algunos registros semióticos como la ecuación lineal, en los estudiantes de noveno grado del colegio Gonzalo Rivera Laguado, evidenciaron errores de sintaxis dificultando el contenido semántico del signo y que afectan la pertinencia del mismo al ser utilizado como recurso semiótico en los procesos argumentativos de situaciones problemas en el campo de las matemáticas.
El problema de la relación sentido-significado de un signo, la no contextualización en el campo de las matemáticas, el aprendizaje mecánico y no significativo, la no apropiación del lenguaje matemático y los errores conceptuales, están dificultando los procesos de argumentación matemática de situaciones problemas, en los estudiantes de noveno grado del colegio Gonzalo Rivera Laguado.

\section{Referencias}

Alcalá, M. La construcción del lenguaje matemático, Barcelona: Graó, 2009.

Ariza, M. (2007). Hacia una representación semiótica de los signos matemáticos, Revista: Mathesis, III, 227-251.

Bachelard, G. La formación del espíritu científico, Buenos Aires: Siglo XXI, 1976.

D’Amore, B. (2006). Objetos, significados, representaciones semióticas y sentido. Revista Latinoamericana de Investigación en Matemática Educativa, Número Especial, 177-195.

Duval, R. Argumentar, demostrar, explicar: ¿continuidad o ruptura cognitiva?, México: Iberoamérica, 1999.

Duval, R. (1999). Algunas cuestiones relativas a la argumentación. La lettre de la Preuve.

Gorlée, D. "La semiótica tríadica de Pierce y su aplicación a los géneros literarios". [En línea]. Disponible en http://www.biblioteca. org.ar/libros/155534.pdf, s/f. ISSN 1794-8231 -ISSN $2462-8794$ PP: $20-32$
Hernández, R. Fernández, C. \& Baptista, P. Metodología de la investigación (6ta edición), México: Mc Graw Hill, (2014). 
No. 1

León, O. \& Calderón, D. La argumentación en matemáticas en el aula: una oportunidad para la diversidad, Bogotá: Universidad Externado de Colombia, 1.996.

León, O. \& Calderón, D. Argumentar y validar en matemáticas ¿una relación necesaria?, Bogotá: Universidad del Valle, 2003.

Marmolejo, G. (2012). Elementos a considerar en la Enseñanza del registro semiótico de las figuras en los primeros ciclos de la educación básica. Revista Ecomatemático, 2(1), 14-19.

Piaget, J. Psicología y pedagogía, Barcelona: Crítica, 2001.

Peirce, C. La ciencia de la semiótica, Recopilación realizada por Hartshorne, C. Weiss, P. y Wiener, P. Buenos aires: Nueva visión, 1974.

Puig, L. (1994). Semiótica y matemáticas. Revista Ectopias, 51, 3-21.

Puig, L. Signos, textos y sistemas matemáticos de signos, México: Universidad de valencia, Ed. En Filloy, 2003.

Vevia, F. Escritos filosóficos charles Sanders Pierce (1er volumen), México: Colegio de Michoacán, 1997.

Vygotsky, L. Pensamiento y lenguaje, Barcelona: Paidós, 1995. 